\title{
COMPARISON OF THE DELTA ROBOT ABB IRB 360 PROPERTIES AFTER COLLISIONS
}

The use of robots with their partial damage is not common, and it is not used in safety terms. If the robot works in a controlled environment after partial damage, the robot can be used for a short period of time when the speed of moving and weight of the load are reduced. In the event of damage one of the arms of the parallel robot, the parallelism of the movable platform towards base is lost. This results in a loss of accuracy, which in the case of shortening one of the robots arms is reflected by a gradual change in the distance of the removal site from the storage site. This change is bigger with the greater distance of the center of gravity effector from the robot output flange. For the short-term unavailability of replacement arms, it was decided to continue the training with the robot with rebuilt and reinforced arm. This article concerned with performing a control measurement to detect the deviation value that could be compensated in the execution of the individual cycles of the robot. The measurement compared values of pose accuracy and pose repeatability of the robot after damage by using measured compensated deviations.

Keywords: delta robot, verification, precision, load, sensors

\section{Introduction}

The basic idea behind the delta robot ABB design is the use of parallelograms. A parallelogram allows an output link to remain at fixed orientation with respect to an input link. The use of three such parallelograms restrain completely the orientation of the mobile platform which remains only with three purely translational degrees of freedom. The input links of the three parallelograms are mounted on rotating levers via revolute joints. The revolute joints of the rotating levers are actuated in two different ways: with rotational (DC or AC servo) motors or with linear actuators. Finally, a fourth leg is used to transmit rotary motion from the base to an end-effector mounted on the mobile platform. The use of base-mounted actuators and low-mass links allows the mobile platform to achieve accelerations of up to $50 \mathrm{G}$ in experimental environments and $12 \mathrm{G}$ in industrial applications. This makes the delta robot $\mathrm{ABB}$ a perfect candidate for pick and place operations of light objects (from $10 \mathrm{~g}$ to $1 \mathrm{~kg}$ ). The delta robots $\mathrm{ABB}$ available on the market operate typically in a cylindrical workspace which is $1 \mathrm{~m}$ in diameter and $0.2 \mathrm{~m}$ high $[1,2]$.

\section{Basic properties of robotized workplace}

The delta robot $\mathrm{ABB}$ is a part of a training workplace equipped with a pair of conveyors. The material flow configuration depends on the type of training that is being realized. At the workplace, it is possible to set two bases of the flow of the material to be handled. In basic training a configuration is used where the delta robot $\mathrm{ABB}$ picks up the manipulated objects from the pallet, which is transported on the Bosch pallet conveyor [3]. During the manipulation, the pallet is placed in a precisely defined position using programmable stops. After removing the object and moving it over the conveyor belt, the manipulated object is released and carried by the conveyor to the respective exit position. In advanced training, conveyor functions are opposite. The delta robot ABB picks up manipulated objects randomly placed on the conveyor belt. It then stores them on selected positions in the technological pallet conveyor Bosch. For manipulation used a vacuum gripper with a suction cup with diameter of 5 to $20 \mathrm{~mm}$. The weight of manipulating objects ranges from $50 \mathrm{~g}$ to $2 \mathrm{~kg}$. $A$ view on robotized workplace equipped with delta robot $A B B$ is shown in Figure 1.

The delta robot $\mathrm{ABB}$ is intended primarily for material handling, part removal, packaging operations or assembly. It is characterized by a high speed of movement, with an integrated

\footnotetext{
* ' 'Jan Semjon, ${ }^{1}$ Marek Sukop, ${ }^{1}$ Marek Vagas, ${ }^{1}$ Rudolf Janos, ${ }^{1}$ Peter Tuleja, ${ }^{1}$ Lucia Koukolova, ${ }^{1}$ Peter Marcinko, ${ }^{1}$ Ondrej Jurus, ${ }^{2}$ Jozef Varga ${ }^{1}$ Department of Robotics, Faculty of Mechanical Engineering, Technical University of Kosice, Slovakia

${ }^{2}$ Prototype and Innovation Center, Faculty of Mechanical Engineering, Technical University of Kosice, Slovakia

Email: jan.semjon@tuke.sk
} 


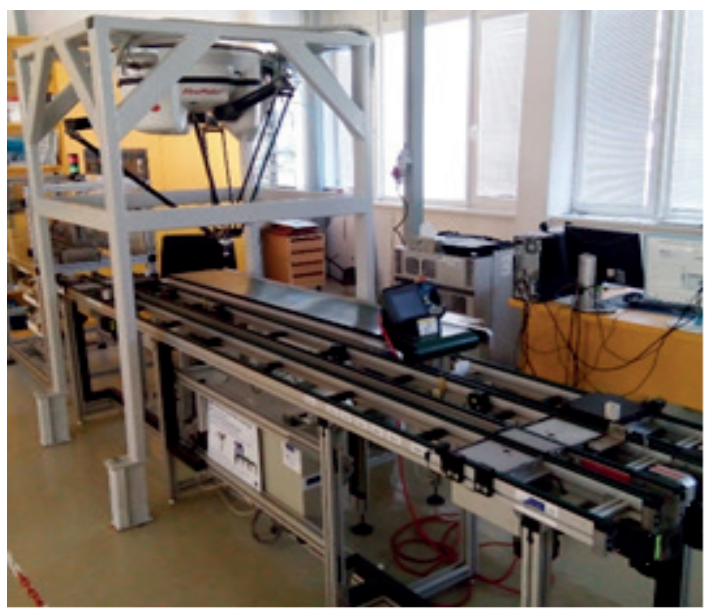

Figure 1 Robotized workplace

vision software and integrated control of indexing belts. Basic parameters of delta robot ABB IRB360-3/1130: (number of degrees of freedom: 4; weight: $120 \mathrm{~kg}$; load capacity: $3 \mathrm{~kg}$; operating radius: $R 565 \mathrm{~mm}$; repeatable positions: $( \pm) 0.1 \mathrm{~mm}$; cycle time: app. 0.52-0.75 s).

During training, there was a collision between one of the robot arms and the RFID chip on the pallet of Bosch pallet conveyor. After the collision, extensive damage to one of the pair of robot arms was detected, resulting in the delta robot ABB training being stopped. After ordering spare parts, it was obvious that the delivery term for the spare arms was 10 days. Due to the necessity of completing the training according to the timetable, we proceeded to temporarily repair this arm. The damaged arm was extensively deformed, we tried to remove the deformation after dismantling. This led to further cracks in the vicinity of the deformation. In order to continue the training, the arm was cut in the crack place. A precision aluminium tube was inserted into the cavity of the carbon fiber arm and with the help of an adhesive connected to the parts of the damaged arm. The surface of the joint was covered by black insulating tape for aesthetic reasons. After the repair was completed, that the difference in length between restrained arm and undamaged arm was less than $0.1 \mathrm{~mm}$. This lead to a slight shift and rotation of the centre of the delta robot ABB output flange during the work cycle. After 50 cycles end of effector towards of the programmed position and the slight deflection of the arm were visibly displaced. Another arm repair was not possible due to the use of glue. After performing further cycles, it was found that the deformation of the arm had stopped and no changes were made [4]. Because of the need to determine the shift value per work cycle, control measurements were taken. The result of the measurement was to find out the displacement value in the Cartesian coordinate system, the value being used to compensate for the inaccuracy caused by the damaged robot arm.

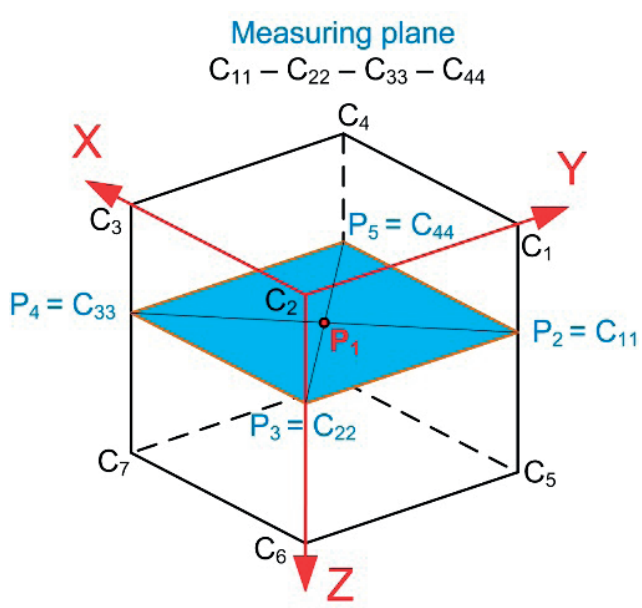

Figure 2 Designed measuring plane

\section{Selection of measurement tests and their description}

After the measurement it was possible to use the measured data to create compensatory deviations. After the implementation of the compensatory deviations in the robot program, control measurements were carried out to compare the identified inaccuracy towards the manufacturer's inaccuracies. To measure the relevance of the measurement carried out, it was based on the data and measurement methodology provided by the delta robot ABB manufacturer. In this materials the manufacturer surveyed robot parameters using ISO $9283[5,6]$, where focused overall on four types of tests (Pose accuracy $A P$, Pose repeatability $R P$ ). The robot manufacturer reports the measured values in Table 1, with speed $0.8 \mathrm{~m} \cdot \mathrm{s}^{-1}$ and a load of $0.1 \mathrm{~kg}$.

In order to compensate for the inaccuracy caused by the damaged arms, we decided to carry out a control measurement using two tests (Pose accuracy AP, Pose repeatability RP). Before the test, the movements of the delta robot ABB must be limited as necessary to set up the measuring instruments. The test must be preceded by a specified heating operation if specified by the manufacturer. The ambient temperature in the tests should be kept within " $20 \pm 2{ }^{\circ} \mathrm{C}$ ". Measured location data (coordinates $x_{j}, y_{j}, z_{j}$ ) must be expressed in a coordinate system whose axes are parallel to the coordinate system axis coordinates of the base of the delta robot ABB being measured. All tests must be carried out under the $100 \%$ (10, $50 \%$ optional) of rated load (mass, centre of gravity, moment of inertia) in accordance with the manufacturer's specification (technical specifications). All position characteristics must be tested at the speed of $100 \%$ (optionally $50 \%, 10 \%$ ) achievable between the prescribed positions. The following requirements must be met placing the measuring cube in the robot workspace: five points (P1 to $\mathrm{P} 5$ ) are located on the diagonals of the selected plane. These five points together with manufacturer's guideline, form the test positions must reach the centre of the mechanical test interface. The test positions must be defined by the coordinates of the base. $\mathrm{P} 1$ is the 
Table 1 Manufacturer's measured values according to ISO 9283

\begin{tabular}{cc}
\hline Characteristic (ISO 9283) & $(\mathrm{mm})$ \\
\hline Pose accuracy (AP) & 0.01 \\
Pose repeatability (RP) & 0.1 \\
\hline
\end{tabular}

diagonal intersection and is the centre of the cube. Points P2 to P5 are spaced from the diagonal ends " $10 \pm 2 \%$ " of the diagonal length. If this is not possible, the nearest diagonal point must be selected. All robots joints must be applied while moving between all test positions. Based on the experiments performed on 100 cycles it was decided that the measurement would not be carried out exactly according to ISO 9283. The robot's workspace has a value " $\pm \mathrm{X}, \mathrm{Y}=342.5 \mathrm{~mm}$ " and “ $\pm \mathrm{Z}=125 \mathrm{~mm}$ ". The dimension of the cube according to the standard is given by the robot's workspace, where it was limited in this case by " $Z=250 \mathrm{~mm}$ ". For this reason, the cube would have a size of $250 \times 250 \times 250 \mathrm{~mm}$, which would not allow a meaningful measurement to be made. The result was a design in which the measurement plane would be parallel to the plane $\mathrm{X}, \mathrm{Y}$ of the delta robot ABB base, Figure 2. The distance between the extreme points of the plane (P2-P3, P3-P4, P4-P5 and P5-P2) is $685 \mathrm{~mm}$. Measuring points P2, P3, $\mathrm{P} 4$ and P5, were selected for measurement where measurements were made to provide similar environmental conditions for all points [7].

Selected test parameters with load definitions, output speed of rotation and cycle counts are listed in Table 2.

Pose accuracy (AP) - represents a deviation between the programmed position and the average of the positions achieved when moving to the programmed position from the same direction. One way position accuracy is calculated according to:

$$
A P_{X}=\left(\bar{x}-x_{C}\right), A P_{Y}=\left(\bar{y}-y_{C}\right), A P_{Z}=\left(\bar{z}-z_{C}\right)
$$

Where, $\left(x_{C}, z_{C}, y_{C}\right)$ are the programmed values and $\left(x, z_{i}, y_{j}\right)$ are the actual (measured) values. While:

$$
\bar{x}=\frac{1}{n} \sum_{j=1}^{n} x_{j}, \bar{y}=\frac{1}{n} \sum_{j=1}^{n} y_{j}, \bar{z}=\frac{1}{n} \sum_{j=1}^{n} z_{j},
$$

The resulting value of one-way wrist precision is based on the relationship:

$$
A P=\sqrt{\left(\bar{x}-x_{C}\right)^{2}+\left(\bar{y}-y_{C}\right)^{2}\left(\bar{z}-z_{C}\right)^{2}}
$$

Pose repeatability (RP) - indicates the degree of match between placements and orientation of the positions achieved after n-repetitions to the same programmed position on the same direction. For a given position, it expresses the RP value that is the radius of the sphere whose centre is the barycentre and which is calculated:

$R P=\bar{l}+3 S_{l}$
Table 2 Selected test parameters

\begin{tabular}{cccc}
\hline Characteristic (ISO 9283) & $\begin{array}{c}\text { Load } \\
(\mathrm{kg})\end{array}$ & $\begin{array}{c}\text { Speed } \\
\left(\mathrm{m}_{\mathrm{s}} \mathrm{s}^{-1}\right)\end{array}$ & Number (cycles) \\
\hline Pose accuracy (AP) & 0.1 & 0.8 & 30 \\
Pose repeatability (RP) & 0.1 & 0.8 & 30 \\
\hline
\end{tabular}

Table 3 Average inaccuracy values on the delta robot ABB, point $P_{1 A}$

\begin{tabular}{cccc}
\hline AP & Axis $\mathrm{X}$ & Axis $\mathrm{Y}$ & Axis $\mathrm{Z}$ \\
\hline Point $\mathrm{P}_{1 \mathrm{~A}}$ & 0.00183 & -0.00171 & 0.00156 \\
\hline
\end{tabular}

where,

$$
\begin{aligned}
& S_{l}=\sqrt{\frac{\sum_{j=1}^{n}\left(l_{j}-\bar{l}\right)}{n-1}}, l=\frac{1}{n} \sum_{j=1}^{n} l, \\
& l_{j}=\sqrt{\left(\bar{x}-x_{C}\right)^{2}+\left(\bar{y}-y_{C}\right)^{2}\left(\bar{z}-z_{C}\right)^{2}}
\end{aligned}
$$

The coordinates of the barycentre of the achieved points at n-repetitions of the same position are calculated according to Equations (2).

A set of three Heidenhain MT 25 sensors was used to make the measurement (system accuracy “ $\pm 2 \mu \mathrm{m}$ ”). The sensors were placed in the measuring base, which ensured their exact fitting in three perpendicular planes. Since the sensors are touching, it is necessary to carry out their admission and removal to the measuring object. This was accomplished by means of a system of three automatic triggers, the control of which was realized by means of control system delta robot ABB. The measured object was a measuring sphere with an outer diameter of $25 \mathrm{~mm}$, with the center located in the axis of the outlet flange. The view of the location of the sensors and the robot is shown in Figure 3.

\section{Results and discussion}

To determine the degree of the accuracy of a robot caused by robot damage, a control measurement was carried out at the four P2 to P5 measurement points. At each point, 30 measurements were made. The average values of the deviation at point P1 were mathematically calculated and graphically verified in CA environments of CREO, Table 3 .

The deviations in the direction of the plane XY axis are shown in Figure 4. Z-axis deviations have been neglected when creating compensation in the respective program. This is due to the use of a spring suction as the end effector that allows the delta robot $\mathrm{ABB}$ in the Z-axis to compensate [8].

The measured deviations were subsequently used in the robot program. On the basis of the tests carried out a method was chosen where, every 5 cycles a half value of the mean deviation was entered in the appropriate direction. After repeating 100 cycles, it was found that the resulting robot inaccuracy was within the prescribed limits. 


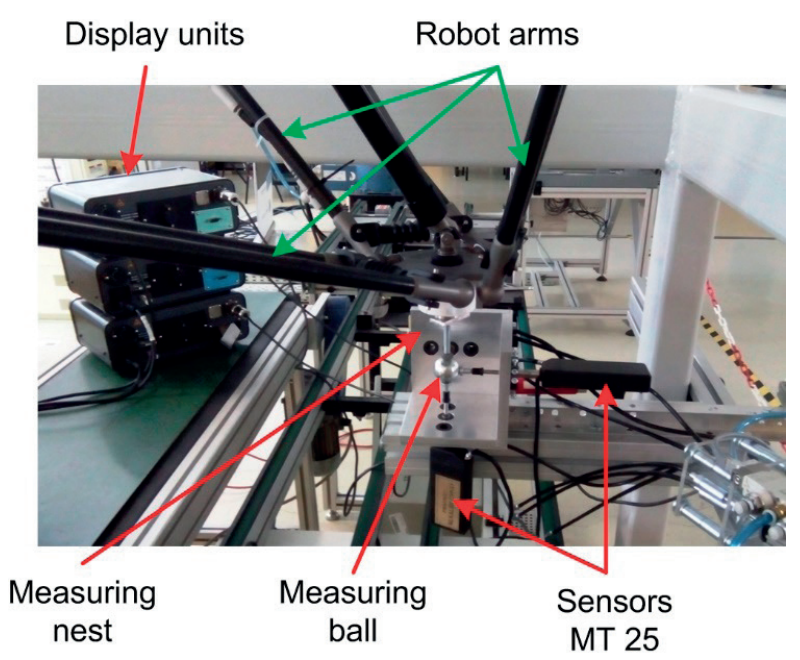

Figure 3 Location of sensors in the robot workspace

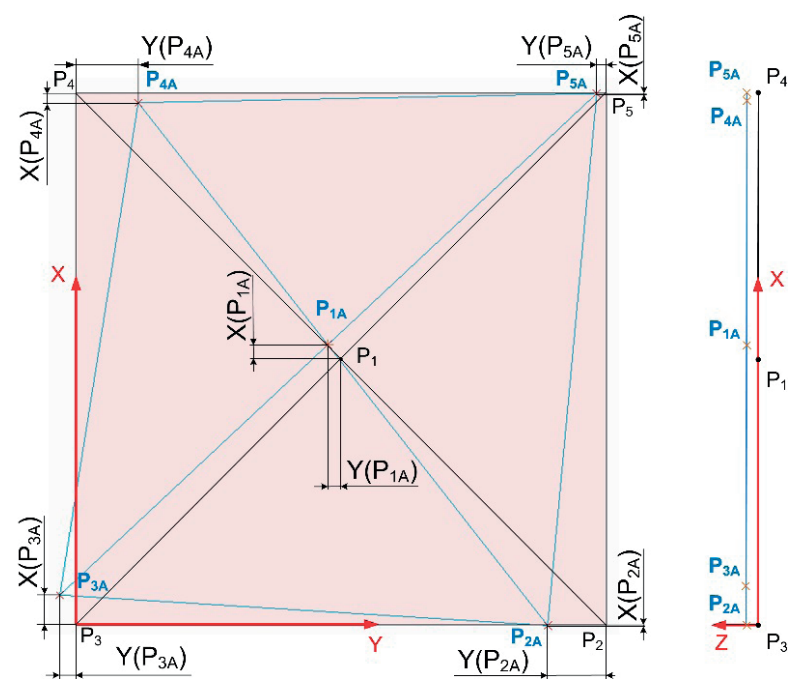

Figure 4 Measured plane $X Y$

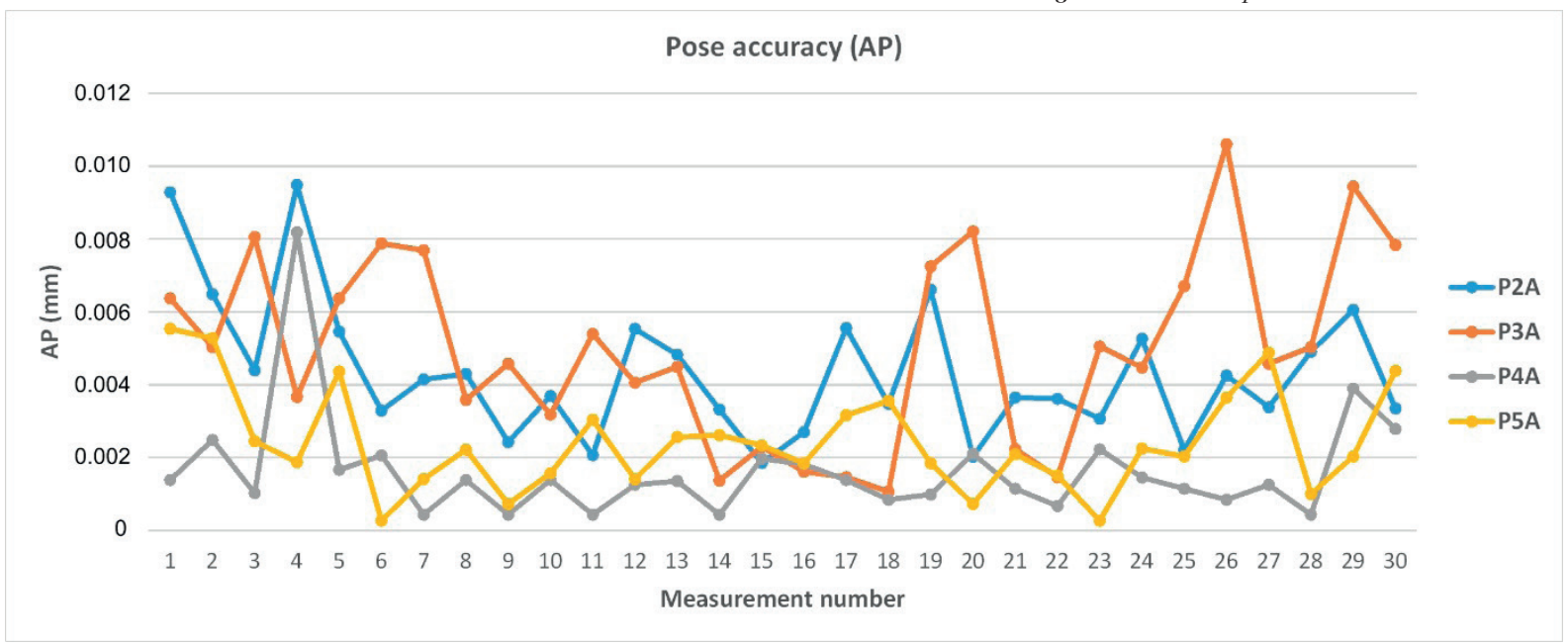

Figure 5 Pose accuracy

After the respective deviations were entered into the robot program a control measurement was performed. The measured values were then processed to determine one-way pose accuracy (AP) and one-way pose repeatability (RP).

Pose accuracy (AP) - Graphical representation of measured data (pose accuracy) in direction the plane $\mathrm{XY}$ is shown in the graph of Figure 5.

After realizing the 30 measurements at $10 \%$ of maximal capacity of delta robot ABB " $0.3 \mathrm{~kg}$ ", we came to the following averages (pose accuracy), Table 4.

Pose repeatability (RP) - Graphical representation of measured data (pose repeatability) in direction the plane $\mathrm{XY}$ is shown in the graph of Figure 6.

After realizing the 30 measurements at $10 \%$ of maximal capacity of delta robot ABB " $0.3 \mathrm{~kg}$ ", we came to the following averages (pose repeatability), Table 5 .

Measurement results have shown that the use of a delta robot $\mathrm{ABB}$ for training purposes is also with arm damage for the necessary time. In a controlled environment where the speed was limited to $1 \mathrm{~m} \cdot \mathrm{s}^{-1}$ and the load per $0.1 \mathrm{~kg}$ it was confirmed for about 500 cycles. Using a damaged robot in a normal running would not be possible in terms of security.

\section{Conclusions}

On the basis of the measured parameter measurements of the wrist-assembled modules with three degrees of freedom, we came to the following conclusions: The partial result of the measurement was the formation of the measured plane whose peaks $\mathrm{P} 2 \mathrm{~A}, \mathrm{P} 3 \mathrm{~A}, \mathrm{P} 4 \mathrm{~A}$ and $\mathrm{P} 5 \mathrm{~A}$ were obtained by determining the mean values of deviations to points $\mathrm{P} 2, \mathrm{P} 3, \mathrm{P} 4$ and $\mathrm{P} 5$. By overlapping the measured plane with the programmed plane it was possible to determine the resulting inaccuracy of the delta robot $\mathrm{ABB}$ at these points. Deviations could be used to create compensation values embedded in the robot's program, so that 


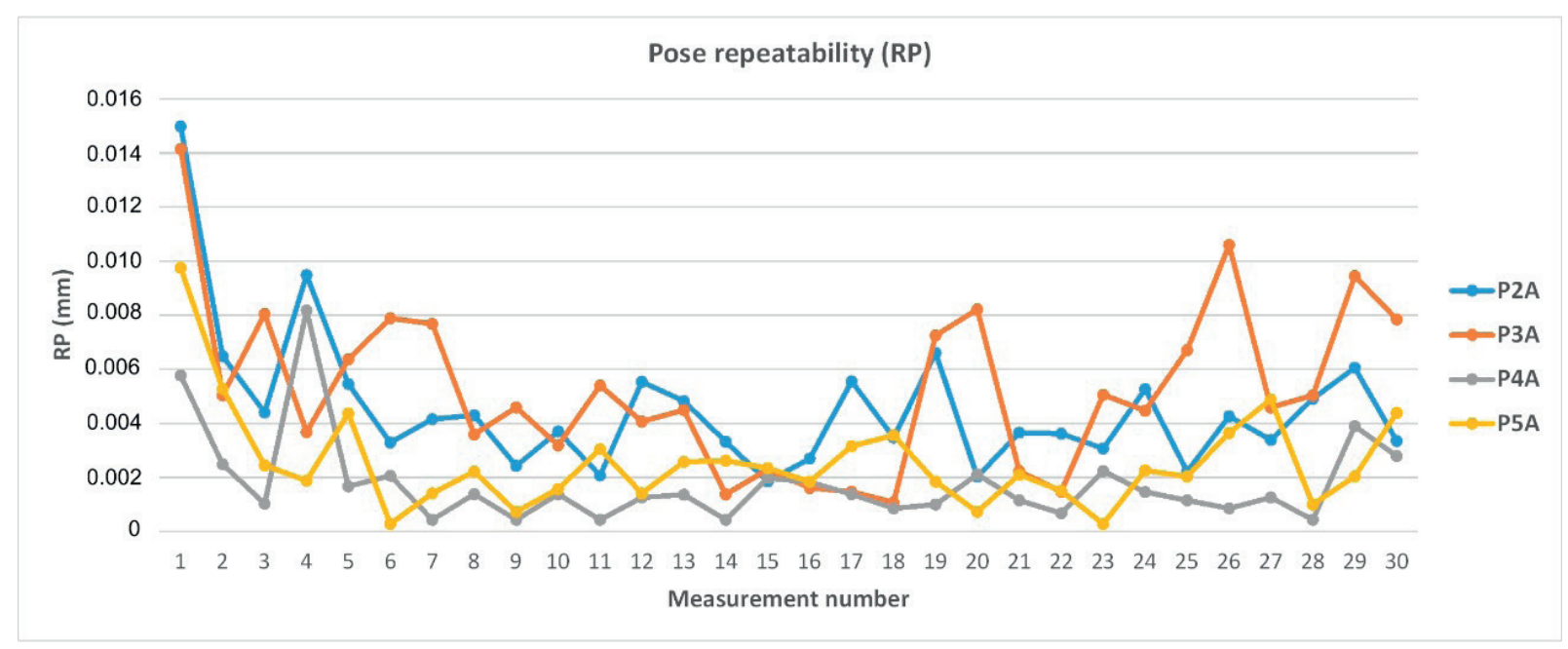

Figure 6 Pose repeatability

Table 4 Average-Pose accuracy (AP)

\begin{tabular}{ccccc}
\hline & $\mathrm{P}_{2}$ & $\mathrm{P}_{3}$ & $\mathrm{P}_{4}$ & $\mathrm{P}_{5}$ \\
\hline $\mathrm{AP}$ & 0.007679 & 0.004818 & $\mathbf{0 . 0 0 8 1 8 4}$ & 0.001573 \\
\hline
\end{tabular}

the resulting robot motion after repeating n-cycles was within the prescribed accuracy.

Based on the delta robot ABB measurement, we have also reached the following conclusions: The manufacturer's prescribed "pose accuracy" at load $0.1 \mathrm{~kg}$ and movement velocity of robot 0.8 $\mathrm{m} . \mathrm{s}^{-1}$ has value $0.01 \mathrm{~mm}$ (Table 4 ) and is higher than the highest average value at the point $\mathrm{P} 4(0.008154 \mathrm{~mm})$. The manufacturer's prescribed "pose repeatability" at load $0.1 \mathrm{~kg}$ and movement velocity of robot $0.8 \mathrm{~m} \cdot \mathrm{s}^{-1}$ has value $0.1 \mathrm{~mm}$ (Table 5) and is higher than the highest average value at the point $\mathrm{P} 3(0.01282 \mathrm{~mm})$. On the basis of realized measurements, we can conclude that of the delta robot $\mathrm{ABB}$ is within the desired range (that is, it meets ISO 9283).
Table 5 Average-Pose repeatability (RP)

\begin{tabular}{ccccc}
\hline & $\mathrm{P}_{2}$ & $\mathrm{P}_{3}$ & $\mathrm{P}_{4}$ & $\mathrm{P}_{5}$ \\
\hline $\mathrm{RP}$ & 0.010058 & $\mathbf{0 . 0 1 2 8 2 0}$ & 0.0060278 & 0.006652 \\
\hline
\end{tabular}

Damaged delta robot ABB from a safety point of view can only be used at lower speeds. For this reason, it is only suitable for use in training or during exhibitions.

\section{Acknowledgements}

This contribution is the result of the project implementation: Research modules for intelligent robotic systems (ITMS: 26220220141), activity 1.2 and 1.3 , supported by the Research \& Development operational Program funded by the ERDF.

\section{References}

[1] Delta Robots ABB [online]. Available: http://www.parallemic.org/Reviews/Review002.html.

[2] Delta Robots ABB [online]. Available: https://library.e.abb.com/public/31305748911b4da18b1463233dd91b83a/3HAC029963-en.pdf.

[3] SHETTY, D., KOLK, R. A.: Mechatronics System Design, SI Version, 2nd edition. Global Engineering, Stanford, 2011.

[4] COP, V., DUROVSKY, F.: (2013). Advanced Mechatronic Components, Driving and Positioning Modules for Intelligent Manufacturing and Robotics Systems. Internationales Forum Mechatronik, Switzerland, 2-19, 2013.

[5] STN EN ISO 9283:2001-12. Manipulating Industrial Robots-Performance Criteria and Related Test Methods. Slovak Office of Standards, Metrology and Testing (SOSMT), Bratislava.

[6] ISO 9283:1998. Manipulating Industrial Robots-Performance Criteria and Related Test Methods. International Organization for Standardization, Geneva.

[7] HOCK, O., DRGONA, P., PASKALA, M.: Simulation Model of Adjustable Arm Using Denavit-Hartenberg Parameters. Proceedings of 10th international conference ELEKTRO 2014, Slovakia, CD-ROM, 176-179, 2014.

[8] HOCK, O., DRGONA, P., FRIVALDSKY, M., HAVRILA, R.: Simulation of Manipulator Positioning Methodology Using Inverse Kinematics. Proceedings of the 16th international scientific conference Electric power engineering (EPE 2015), Czech Republic, 706-711, 2015. 